Part of Journal of Research of the National Bureau of Standards, Volume 20, January 1938

\title{
APPROXIMATE GLASS CONTENT OF COMMERCIAL PORTLAND CEMENT CLINKER
}

\author{
By William Lerch ${ }^{1}$
}

\section{ABSTRACT}

The approximate glass content was determined, by the heat of solution method, for commercial portland cement clinker obtained from 21 plants throughout the United States. The approximate glass content of these commercial clinker compositions was found to vary from 2 to 21 percent. The approximate glass content was also determined for these clinker compositions after the clinker had been subjected to special heat treatment in the laboratory. The results indicate that for a given clinker composition, the heat of solution of the clinker, and likewise the glass content, are dependent on the cooling conditions to which the clinker has been subjected. Relatively high glass contents may be obtained by cooling the clinker rapidly, and relatively low glass contents by cooling it slowly.

\section{CONTENTS}

I. Introduction

II. Experimental procedure

1. Treatment of the clinker

2. Determination of the approximate glass content of the clinker-

3. Estimation of the crystalline $\mathrm{MgO}$ in the clinker

\section{INTRODUCTION}

Numerous attempts to correlate the properties of commercial portland cements with their oxide composition or computed compound composition, have met with only partial success. Cements of almost identical composition, and ground to the same degree of fineness, have shown pronounced differences in physical properties.

These variations in properties are not due to differences in chemical composition but to some other cause, for instance the heat treatments to which the clinker has been subjected during or following clinkering.

At the clinkering temperatures attained by present-day methods of portland cement manufacture, a considerable part of the charge is present as a liquid phase, the percentage of liquid produced being dependent on the chemical composition of the charge and on the maximum temperature attained. The disposition of this liquid phase in the clinker will be dependent on the subsequent rate of cooling. If the cooling process is sufficiently rapid, a condition may be approached in which the liquid phase will be present as an "undercooled

\footnotetext{
1 Research Associate at the National Bureau of Standards, representing the Portland Cement Association Fellowship.
} 
liquid" or glass. But if the cooling process is sufficiently slow to permit a continuous equilibrium, a completely crystalline product may be obtained. Intermediate rates of cooling may produce clinker of some degree of crystallization intermediate between these limits. The conditions of kiln operation which may be expected to affect the degree of crystallization, or conversely the glass content of the clinker are; the maximum kiln temperature; the rate at which the clinker passes through the kiln; the position of the hot zone with respect to the discharge end of the kiln; and the schedule of clinker cooling employed within the kiln and immediately subsequent to discharge from the kiln.

In a previous investigation by Lerch and Taylor [1] ${ }^{2}$ it was found that the heat treatment of portland cement clinker has an important effect on some of the properties of the cement. The heat treatments employed in that study were of such character that different degrees of crystallization of the cement compounds and consequently different glass content might be anticipated, though the glass content was not determined quantitatively. A recent report by Lerch and Brownmiller [2] describes a heat of solution method for obtaining the approximate glass content of portland cement clinker. In the latter investigation it was found that rapidly cooled clinkers, prepared in an experimental kiln from commercial raw materials, contained relatively large quantities of glass.

The present report describes the results obtained for the approximate glass content, as determined by the heat of solution method, of 21 samples of standard portland cement clinker obtained from different plants throughout the United States as well as one additional sample of sulfate-resistant cement clinker. This report also contains the results obtained for the approximate glass content of these clinker compositions after they had been subjected to special heat treatments in the laboratory. The special heat treatments, to which the clinker was subjected in the laboratory, were of such character that different degrees of crystallization of the clinker compounds, consequently differences in the glass content, might be anticipated.

\section{EXPERIMENTAL PROCEDURE}

\section{TREATMENT OF THE CLINKER}

In the request to the plants which supplied the clinker, it was suggested that the clinker should be freshly burned, should not be waterquenched, and should not have been exposed to undue moisture on the stock pile. As a result of this request the samples, as received from the plants, were all of relatively low ignition loss.

Each clinker sample, as received, was crushed between rolls, thoroughly mixed, and divided into three lots, one lot to be used as received, one lot to be reburned and rapidly cooled, and one lot to be reburned and slowly cooled. These samples were prepared and reburned in laboratory kilns by W. C. Taylor following methods described in a previous report [1]. The clinker to be rapidly cooled was reburned in an experimental rotary kiln at $1,400^{\circ} \mathrm{C}$. The clinker obtained from this kiln was small, not exceeding 0.5 inch in diameter, and in the operation of the kiln the clinker cooled from the clinkering tem-

\footnotetext{
${ }^{2}$ Numbers in brackets refer to literature references at the end of this paper.
} 
perature to that of a dull-red heat, about $1,000^{\circ} \mathrm{C}$, in 2 or 3 minutes. This clinker was designated "rapidly cooled clinker." The clinker to be slowly cooled was placed in an updraft gas-fired kiln and heated to $1,400^{\circ} \mathrm{C}$. The gas and air were then adjusted in such manner as to allow the temperature of the clinker to decrease slowly over a 2hour period to $1,250^{\circ} \mathrm{C}$. The gas and air were then cut off and the clinker was allowed to cool with the kiln. This clinker was designated "slowly cooled clinker." The third lot of clinker was used as received from the plant and was designated "plant clinker."

\section{DETERMINATION OF THE APPROXIMATE GLASS CONTENT OF THE CLINKER}

The determination of the approximate glass content of portland cement clinker, by the heat of solution method, is dependent on the latent heat of crystallization of the liquid phase. Thus it becomes necessary to prepare a crystalline material of each clinker somple. The crystallized materials were prepared by carefully "annealing" 3 a small quantity of the plant clinker in an electric furnace. The samples were placed in the furnace and heated to $1,400^{\circ} \mathrm{C}$. The temperature of the furnace was caused to drop slowly over a period of 2 hours to $1,250^{\circ} \mathrm{C}$, which is slightly below the temperature of final solidification of the liquid, and the charge was held at that temperature $\pm 10^{\circ} \mathrm{C}$ for 15 hours.

The heats of solution were determined, by the use of a vacuum-flask calorimeter [3], for the rapidly' cooled clinker, the slowly cooled clinker, the plant clinker and the crystalline material obtained by "annealing" in the electric furnace. The heats of solution were determined in duplicate and the duplicate determinations agreed to within $3 \mathrm{cal} / \mathrm{g}$ in every case.

Table 1 gives the complete data for all of the clinker samples used in this investigation, including the partial oxide composition, the calculated compound composition as computed by Bogue [4] and Dahl [5], the $\mathrm{Al}_{2} \mathrm{O}_{3} / \mathrm{Fe}_{2} \mathrm{O}_{3}$ ratio, the heats of solution on the ignited weight basis, and the approximate glass content of the clinker.

The clinker compositions which were "annealed" in the electric furnace and those which were slowly cooled in the updraft gas-fired kiln showed various degrees of dusting. Complete dusting occurred with some samples when "annealed" in the electric furnace and partial dusting occurred in some of the slowly cooled samples. There was no evidence of dusting with any of the plant clinker or any of the clinker which was rapidly cooled.

Since dusting, which is the result of beta to gamma inversion of $2 \mathrm{CaO} . \mathrm{SiO}_{2}$, involves a thermal change, it was necessary to make a correction for this thermal change before calculating the approximate glass content of the clinker. Johannson and Thorvaldson. [6] found that the heat involved in the transition of $2 \mathrm{CaO} \mathrm{SiO}_{2}$ from the beta to gamma modification was $6.0 \mathrm{cal} / \mathrm{g}$ at $20^{\circ} \mathrm{C}$. Thus for the compositions which dusted completely, when annealed in the electric furnace, a correction of $0.06 \mathrm{cal} / \mathrm{g}$ for each 1 percent of $2 \mathrm{CaO} . \mathrm{SiO}_{2}$ was added to the obtained heats of solution. For the annealed compositions which

3 The term "annealing", as used in this report, involves a slow cooling process which might be expected to produce a maximum degree of crystallization.

$32208-38-6$ 
showed only slight dusting or no dusting, no corrections were applied to the obtained heats of solution.

The difference between the heat of solution of a given lot of clinker and the corrected heat of solution of the same clinker "annealed" in the electric furnace constitutes the basis for calculating the approximate glass content for the clinker [2]. It is shown in table 1 that these differences are always positive for the plant clinker and for the rapidly cooled clinker, but that they are sometimes negative for the slowly cooled clinker. These negative values obtained with the slowly cooled clinker may be accounted for as a result of small errors in the determination of the heats of solution, and as a result of thermal changes involved in the partial inversion of beta to gamma $2 \mathrm{CaO} . \mathrm{SiO}_{2}$ in some of the slowly cooled samples.

\section{ESTIMATION OF THE CRYSTALLINE MgO IN THE CLINKER}

In making the determinations of heats of solution it was found that periclase, crystalline $\mathrm{MgO}$, as present in the clinker dissolved slowly in the acid solution. This suggested the possibility of a separation of $\mathrm{MgO}$ by the solution method. The sample of ground clinker was introduced into the calorimeter in the usual manner and the stirring continued for 10 minutes. The solution was then filtered on a suction filter and the residue ignited and weighed. By microscopic examination it was observed that these insoluble residues consisted almost entirely of periclase, with not more than a trace of other material present. It was found that for a clinker of low $\mathrm{MgO}$ content, less than 2.0 percent, this insoluble residue would be very low regardless of the cooling conditions to which the clinker had been subjected. With a clinker of higher $\mathrm{MgO}$ content, 2.4 percent or more, a larger insoluble residue was obtained by this method, and the quantity of insoluble residue varied with each clinker composition, depending on the method of cooling the clinker. A larger insoluble residue was obtained from the slowly cooled clinker than that obtained from the rapidly cooled clinker of the same composition. This seems to give further evidence that in the rapidly cooled clinker part of the $\mathrm{MgO}$ was dissolved in the glass with the result that this clinker contained less crystalline $\mathrm{MgO}$, periclase, than did the corresponding slowly cooled clinker. The results of these determinations of insoluble $\mathrm{MgO}$ are given in table 1. It should be borne in mind that these results do not represent the quantity of periclase present in the clinker, since part of the periclase has dissolved during the 10-minute stirring interval.

\section{DISCUSSION}

The clinker samples used in this investigation were obtained from 21 plants throughout the United States ; the range of their oxide composition represents the approximate range of the clinker compositions used in the manufacture of istandard portland cement at the present time. It also seems probable that the methods of manufacture employed at these various plants constitute a representative cross section of present-day manufacturing processes.

The approximate glass content of the plant clinker was determined by the heat of solution method. The approximate glass content was also determined for these clinker compositions after they had been 


\begin{tabular}{|c|c|c|c|c|c|c|c|c|c|c|c|c|c|c|c|c|c|c|c|c|c|c|c|c|c|c|c|c|c|c|c|c|c|}
\hline \multirow{3}{*}{\multicolumn{2}{|c|}{ Clinker ${ }^{1}$}} & \multicolumn{9}{|c|}{ Chemical composition 2} & \multicolumn{5}{|c|}{ Major constituents, corrected } & \multicolumn{4}{|c|}{ Additional minor constituents } & \multicolumn{6}{|c|}{ Calculated compound composition } & \multirow{3}{*}{$\begin{array}{l}\mathrm{Al}_{2} \mathrm{O}_{3} / \\
\mathrm{Fe}_{2} \mathrm{O}_{3}\end{array}$} & \multicolumn{4}{|c|}{ Heat of solution-ignited basis ? } & \multirow{3}{*}{$\begin{array}{c}\text { Ap- } \\
\text { proxi- } \\
\text { mate } \\
\text { glass } \\
\text { content }\end{array}$} & \multirow{3}{*}{$\begin{array}{c}\text { Mgo } \\
\text { remain- } \\
\text { ing in- } \\
\text { soluble } \\
\text { at 10 } \\
\text { min }\end{array}$} & \multirow{3}{*}{$\underset{\text { ker } 1-}{\text { Clink- }}$} \\
\hline & & \multirow[b]{2}{*}{$\mathrm{SiO}_{2}$} & \multirow{2}{*}{$\mathrm{Fe}_{2} \mathrm{O}_{3}$} & \multirow{2}{*}{$\mathrm{Al}_{2} \mathrm{O}_{3}$} & \multirow{2}{*}{$\mathrm{CaO}$} & \multirow{2}{*}{$\mathrm{MgO}$} & \multirow{2}{*}{$\mathrm{SO}_{3}$} & \multirow{2}{*}{$\begin{array}{l}\text { Loss } \\
\text { on ig. } \\
\text { nition }\end{array}$} & \multirow{2}{*}{$\begin{array}{c}\text { Insol- } \\
\text { uble } \\
\text { residue }\end{array}$} & \multirow{2}{*}{$\begin{array}{l}\text { Free } \\
\text { CaOO }\end{array}$} & \multirow{2}{*}{$\mathrm{SiO}_{2}$} & & & CaO, & & & & & & & & & & & & & & & & & & & \\
\hline & & & & & & & & & & & & $\begin{array}{l}\mathrm{FeO} \text { as } \\
\mathrm{Fe}_{2} \mathrm{O}_{3}\end{array}$ & $\begin{array}{l}\mathrm{Tiss}_{2} \\
\mathrm{TiO}_{2}\end{array}$ & $\begin{array}{l}\text { free } \\
\mathrm{CaOO}\end{array}$ & $\operatorname{Mg} 0$ & reo & 1108 & Nayo & $\mathrm{K}_{2} \mathrm{O}$ & 430 & 020 & C/A & C & $\mathrm{MgO}$ & $\mathrm{CaO}$ & & $\begin{array}{c}\text { Ob. } \\
\text { tained } \\
\mathrm{i}\end{array}$ & $\begin{array}{c}\text { Corrected } \\
\text { for C2 } \\
\text { inversion }\end{array}$ & tained) & & & & \\
\hline & $\begin{array}{l}13721 \\
137222 \\
0.27202\end{array}$ & $\begin{array}{l}\% \\
21.5\end{array}$ & $\begin{array}{c}\% \\
2.2\end{array}$ & $\begin{array}{l}\% \\
6.3\end{array}$ & $\begin{array}{l}\% \\
64.3\end{array}$ & $\begin{array}{c}\% \\
3.0\end{array}$ & $\begin{array}{l}\% \\
0.7\end{array}$ & $\begin{array}{l}\% \\
0.4\end{array}$ & $\begin{array}{c}\% \\
0.2\end{array}$ & & $\begin{array}{l}\% \\
21.5\end{array}$ & $\begin{array}{c}\% \\
2.2\end{array}$ & $\begin{array}{c}\% \\
6.0\end{array}$ & $\begin{array}{l}\% \\
63.1\end{array}$ & $\begin{array}{l}\% \\
3.0\end{array}$ & $\begin{array}{r}\% \\
0\end{array}$ & $\begin{array}{c}\% \\
0.3\end{array}$ & \begin{tabular}{c||}
$\%$ \\
0.1
\end{tabular} & \begin{tabular}{c|}
$\%$ \\
1.6
\end{tabular} & $\begin{array}{l}\% \\
50.0\end{array}$ & \begin{tabular}{l|}
$\%$ \\
24.0
\end{tabular} & $\begin{array}{l}\% \\
12.2\end{array}$ & $\begin{array}{c}\% \\
6.7\end{array}$ & $\begin{array}{c}\% \\
3.0\end{array}$ & \begin{tabular}{c|}
$\%$ \\
1.2
\end{tabular} & 2.9 & $\begin{array}{c}\mathrm{cal} / \mathrm{g} \\
642.3\end{array}$ & $\begin{array}{c}\text { callo } \\
643.8\end{array}$ & $\left\{\begin{array}{c}\text { callg } \\
642.6 \\
64.8\end{array}\right.$ & $\begin{array}{l}c \text { callo } \\
-1.2 \\
+1.0\end{array}$ & $\begin{array}{rr}\%_{2} & \\
2 & \\
2\end{array}$ & \begin{tabular}{r|r}
$\%_{0.8}$ \\
3 \\
3
\end{tabular} & $\begin{array}{l}\text { iS } \\
\text { 1P }\end{array}$ \\
\hline $\begin{array}{l}1 \mathrm{Q} \\
2 \mathrm{P} \\
2 \mathrm{P} \\
2 \mathrm{Q}\end{array}$ & $\begin{array}{l}13723 \\
13724 \\
13725 \\
13726 \\
13727\end{array}$ & 20.6 & 5.7 & 6.0 & 64.1 & 1.8 & .6 & .2 & .2 & $\begin{array}{r}1.5 \\
0.1 \\
.8 \\
.9\end{array}$ & 20.6 & 5.5 & 5.6 & 63.3 & 1.8 & .2 & .4 & .3 & 0.7 & 55.5 & 17.2 & 5.6 & 16.7 & 1.8 & 0.8 & 1.1 & 627.3 & 628.5 & $\begin{array}{l}668.0 \\
629.1 \\
630.6 \\
637.4\end{array}$ & $\begin{array}{l}4.2 \\
0.6 \\
2.1 \\
8.9\end{array}$ & $\begin{array}{r}8 \\
1 \\
5 \\
21\end{array}$ & $\begin{array}{l}.2 \\
.1 \\
.1 \\
.1\end{array}$ & $\begin{array}{l}1 \mathrm{Q} \\
2 \mathrm{~S} \\
2 \mathrm{P} \\
2 \mathrm{Q} \\
2 \mathrm{Q}\end{array}$ \\
\hline $\begin{array}{l}3 \mathrm{~S} \\
3 \mathrm{P} \\
3 \mathrm{Q}\end{array}$ & $\begin{array}{l}137727 \\
13728 \\
137729 \\
13720\end{array}$ & 22.4 & 2.7 & 5.9 & 63.9 & 4.0 & .1 & .3 & .2 & & 22.4 & 2.3 & 5.4 & 63.5 & 4.0 & .4 & .5 & .6 & .2 & 48.5 & 27.7 & 10.4 & 7.0 & 4. 0 & .4 & 2.2 & 637.3 & 639.1 & $\begin{array}{l}658.5 \\
644.4 \\
647.2 \\
623.2\end{array}$ & $\begin{array}{r}-0.6 \\
+5.3 \\
8.1\end{array}$ & $\begin{array}{l}0 \\
11 \\
16\end{array}$ & $\begin{array}{l}1.1 \\
0.6 \\
0.5 \\
1.3\end{array}$ & \begin{tabular}{|l}
$5 \mathrm{~S}$ \\
$3 \mathrm{P}$ \\
$3 \mathrm{Q}$
\end{tabular} \\
\hline $\begin{array}{l}4 \mathrm{4} \\
4 \mathrm{P} \\
4 \mathrm{Q}\end{array}$ & $\begin{array}{l}13730 \\
13731 \\
13732\end{array}$ & 19.8 & 7.0 & 5.1 & 62.7 & 4.2 & .1 & .2 & .4 & $\begin{array}{l}4.3 \\
1.6 \\
1.6\end{array}$ & 19.8 & 5.3 & 4.7 & 58.5 & 4. 2 & 1.5 & .4 & .5 & .2 & 48.3 & 20.3 & 3.5 & 16.1 & 4.2 & 4.2 & 0.8 & 632.6 & 632.6 & $\begin{array}{l}683.2 \\
641.1 \\
641.7\end{array}$ & $\begin{array}{l}8.5 \\
9.6\end{array}$ & $\begin{array}{l}21 \\
22\end{array}$ & $\begin{array}{c}1.3 \\
0.6 \\
.5\end{array}$ & $\begin{array}{l}4 \mathrm{~s} \\
4 \mathrm{P} \\
4 \mathrm{Q}\end{array}$ \\
\hline $\begin{array}{l}6 \mathrm{~S} \\
6 \mathrm{P} \\
6 \mathrm{Q} \\
6 \mathrm{Q}\end{array}$ & $\begin{array}{l}13736 \\
13737 \\
13738 \\
30708\end{array}$ & 21.7 & 3.1 & 6. 2 & 66.6 & 1.4 & .3 & .2 & .2 & & 21.7 & 3,1 & 5.9 & 66.2 & 1.4 & 0 & .3 & .1 & .1 & 60.5 & 16.7 & 10.4 & 9.4 & 1.4 & 0.4 & 2.0 & 638.7 & 638.7 & $\begin{array}{l}670.3 \\
639.5 \\
645.5 \\
645.5\end{array}$ & $\begin{array}{l}1.0 \\
0.8 \\
6.8 \\
0.8\end{array}$ & $\begin{array}{r}3 \\
2 \\
14 \\
2\end{array}$ & $\begin{array}{l}.1 \\
.1 \\
.1\end{array}$ & $\begin{array}{l}6 \mathrm{~S} \\
6 \mathrm{P} \\
6 \mathrm{Q}\end{array}$ \\
\hline $\begin{array}{l}7 \mathrm{f} \\
7 \mathrm{P} \\
7 \mathrm{PQ}\end{array}$ & $\begin{array}{l}137399 \\
13740 \\
13741 \\
3749\end{array}$ & 21.1 & 2.4 & 7.2 & 64.2 & 3.4 & .4 & .2 & .2 & ${ }_{4}^{{ }_{4}^{4}}$ & 21.1 & 2.4 & 6.9 & 63.8 & 3.4 & tr & .3 & .4 & .6 & 49.6 & 23.1 & 14.2 & 7.3 & 3.4 & .4 & 3.0 & 645.1 & 645.1 & $\begin{array}{l}649.3 \\
655.5 \\
637.8\end{array}$ & $\begin{array}{r}4.2 \\
10.4 \\
-0.6\end{array}$ & $\begin{array}{r}3 \\
21 \\
21\end{array}$ & $\begin{array}{l}.4 \\
: 2 \\
.7\end{array}$ & $\begin{array}{l}7 \mathrm{P} \\
7 \mathrm{Q} \\
3 \mathrm{~S}\end{array}$ \\
\hline $\begin{array}{l}85 \\
8 \mathrm{PQ} \\
8 \mathrm{Q}\end{array}$ & $\begin{array}{l}13742 \\
13743 \\
13744\end{array}$ & 22.5 & 3.0 & 5.3 & 64.5 & 3.5 & .3 & .3 & .1 & .6 & 22.5 & 3.0 & 4.9 & 63.9 & 3.5 & 0 & .4 & .2 & .5 & 51.9 & 25.4 & 8.0 & 9.1 & 3.5 & .6 & 1.8 & 636.8 & 638.4 & $\begin{array}{l}643.5 \\
644.2 \\
637.2 \\
637.4\end{array}$ & $\begin{array}{r}+5.1 \\
5.8 \\
0.1\end{array}$ & $\begin{array}{l}11 \\
12 \\
0\end{array}$ & $\begin{array}{r}4 \\
1.4 \\
1.0\end{array}$ & \begin{tabular}{|l|l|}
$8 P$ \\
$8 Q$ \\
$9 S$
\end{tabular} \\
\hline 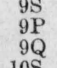 & $\begin{array}{l}13745 \\
13746 \\
13747 \\
1374\end{array}$ & 22.7 & 2.2 & 5.9 & 63.6 & 4.2 & .3 & .3 & .3 & $\begin{array}{l}.8 \\
.8 \\
.9\end{array}$ & 22.7 & 2.2 & 5.6 & 62.8 & 4. 2 & 0 & .3 & .4 & .5 & 42.3 & 33.1 & 11.1 & 6.7 & 4. 2 & .8 & 2.7 & 635.2 & 637.3 & $\begin{array}{l}60.7 \\
641.5 \\
644.1 \\
672.1\end{array}$ & $\begin{array}{l}0.1 \\
4.2 \\
6.8\end{array}$ & \begin{tabular}{r|r}
8 \\
14 \\
14
\end{tabular} & $\begin{array}{r}0.4 \\
.3 \\
.3\end{array}$ & $\begin{array}{l}\text { 9P } \\
9 \mathrm{P} \\
9 \mathrm{Q}\end{array}$ \\
\hline $\begin{array}{l}10 \mathrm{~S} \\
10 \mathrm{P} \\
10 \mathrm{Q} \\
11 \mathrm{~S}\end{array}$ & $\begin{array}{l}13748 \\
13799 \\
13750 \\
1375\end{array}$ & 23.5 & 2.9 & 5.1 & 65.0 & 1.3 & .6 & .5 & .2 & $\begin{array}{l}2.2 \\
2.0 \\
0.1\end{array}$ & 23.5 & 2.9 & 4.8 & 62.9 & 1.3 & 0 & .3 & .3 & .8 & 41.0 & 36.5 & 7.8 & 8.8 & 1.3 & 2.1 & 1.8 & 621.0 & 623.3 & $\begin{array}{l}62.8 \\
624.1 \\
628.2 \\
630.5\end{array}$ & $\begin{array}{l}0.5 \\
4.9 \\
1.9\end{array}$ & $\begin{array}{r}1 \\
2 \\
10 \\
3\end{array}$ & $\begin{array}{l}.1 \\
.1 \\
.1 \\
1\end{array}$ & $\begin{array}{l}10 \mathrm{P} \\
10 \mathrm{Q} \\
11 \mathrm{~S}\end{array}$ \\
\hline $\begin{array}{l}118 \\
110 \\
110\end{array}$ & $\begin{array}{l}13751 \\
13752 \\
13753 \\
13753\end{array}$ & 220 & 5.1 & 4.9 & 66.3 & 0.8 & .1 & .2 & .2 & $\begin{array}{l}0.1 \\
.3 \\
.3\end{array}$ & 22.0 & 5.1 & 4. 6 & 66.1 & 0.8 & 0 & .3 & .7 & .2 & 63.7 & 15.0 & 3.6 & 15.5 & 0.8 & 0.2 & 1.0 & 629.4 & 629.4 & $\begin{array}{l}630.5 \\
6322.3 \\
6322.7 \\
632.7\end{array}$ & $\begin{array}{l}1.1 \\
2.9 \\
3.3\end{array}$ & $\begin{array}{l}3 \\
7 \\
8 \\
1\end{array}$ & .1 1 & $\begin{array}{l}115 \\
110 \\
110\end{array}$ \\
\hline $\begin{array}{l}12 \mathrm{~S} \\
12 \mathrm{P} \\
12 \mathrm{Q}\end{array}$ & $\begin{array}{l}13754 \\
13755 \\
13756 \\
13756\end{array}$ & 22.0 & 6.2 & 5.2 & 63.1 & 2.4 & .2 & .3 & .3 & ${ }_{t r}^{0}$ & 22.0 & 6.2 & 4.9 & 63.1 & 2.4 & $\operatorname{tr}$ & .3 & .3 & .4 & 47.9 & 27.0 & 2.5 & 18.9 & 2.4 & $\operatorname{tr}$ & 0.8 & 619.1 & 619.1 & $\begin{array}{l}619.5 \\
623.1 \\
627.7 \\
646.8\end{array}$ & $\begin{array}{r}0.4 \\
4.0 \\
8.6 \\
-1.2\end{array}$ & $\begin{array}{r}1 \\
9 \\
21 \\
0\end{array}$ & $\begin{array}{r}.6 \\
.2 \\
1.2 \\
1.2\end{array}$ & $\begin{array}{l}12 \mathrm{P} \\
12 \mathrm{P} \\
12 \mathrm{Q} \\
13 \mathrm{~S}\end{array}$ \\
\hline $\begin{array}{l}138 \\
13 \mathrm{P} \\
13 \mathrm{Q}\end{array}$ & $\begin{array}{l}1360 \\
13758 \\
13759 \\
13759\end{array}$ & 21.9 & 2.7 & 5.8 & 64.1 & 4.3 & .4 & .2 & .2 & $\begin{array}{l}: 4 \\
: 6 \\
6 \\
\end{array}$ & 21.9 & 2.7 & 5.4 & 63.7 & 4. 3 & 0 & .4 & .2 & .6 & 52.7 & 23.1 & 9.8 & 8.2 & 4. 3 & 0.4 & 2.1 & 648.0 & $6: 8.0$ & $\begin{array}{l}649.8 \\
649.8 \\
655.2 \\
646.5\end{array}$ & $\begin{array}{r}+1.8 \\
7.2 \\
-0.7\end{array}$ & $\begin{array}{r}4 \\
15 \\
0\end{array}$ & $\begin{array}{l}0.7 \\
.5 \\
.0\end{array}$ & $\begin{array}{l}13 \mathrm{P} \\
13 \mathrm{Q} \\
134\end{array}$ \\
\hline $\begin{array}{l}14 \mathrm{~S} \\
14 \mathrm{P} \\
14 \mathrm{Q}\end{array}$ & $\begin{array}{l}13760 \\
13761 \\
13762 \\
3_{3}^{3} 762\end{array}$ & 21.9 & 2.5 & 5.3 & 64.2 & 3. 9 & .5 & .4 & .3 & $\begin{array}{l}\mathrm{tr} \\
.9 \\
\end{array}$ & 21.9 & 2.5 & 5.0 & 63.3 & 3. 9 & 0 & .3 & .3 & 1.0 & 54.0 & 22.0 & 9.1 & 7.6 & 3.9 & .9 & 2.1 & 645.8 & 647. 2 & $\begin{array}{l}6+0.0 \\
650.4 \\
654.7 \\
636.7\end{array}$ & $\begin{array}{r}-2.8 \\
7.5 \\
1.0\end{array}$ & \begin{tabular}{r|}
6 \\
15 \\
2
\end{tabular} & $\begin{array}{l}0.5 \\
.4 \\
1.3\end{array}$ & $\begin{array}{l}14 \mathrm{P} \\
14 \mathrm{P} \\
15 \mathrm{Q} \\
15 \mathrm{~S}\end{array}$ \\
\hline $\begin{array}{l}158 \\
15 \mathrm{P} \\
15 \mathrm{Q}\end{array}$ & $\begin{array}{l}13773 \\
1374 \\
13765 \\
13765\end{array}$ & 21.8 & 3. 0 & 5.7 & 63.4 & 4. 2 & .6 & .3 & .1 & $\begin{array}{l}1.1 \\
1.4 \\
1.4\end{array}$ & 21.8 & 3.0 & 5.4 & 62.1 & 4. 2 & 0 & .3 & .3 & 0.9 & 46.6 & 27.4 & 9.2 & 9.1 & 4. 2 & 1.3 & 1.9 & 635.7 & 635.7 & $\begin{array}{l}638.0 \\
6343.7 \\
643.7 \\
643\end{array}$ & $\begin{array}{r}2.3 \\
8.0 \\
-1.2\end{array}$ & $\begin{array}{r}5 \\
16 \\
0\end{array}$ & $\begin{array}{r}0.6 \\
.5 \\
5\end{array}$ & \begin{tabular}{|l}
$15 \mathrm{P}$ \\
$15 \mathrm{Q}$ \\
$116 \mathrm{~S}$
\end{tabular} \\
\hline $\begin{array}{l}16 \mathrm{~S} \\
16 \mathrm{P} \\
16 \mathrm{Q} \\
17 \mathrm{~s}\end{array}$ & $\begin{array}{l}13766 \\
13767 \\
13768 \\
13760\end{array}$ & 22.3 & 2.6 & 6.3 & 65.0 & 3.3 & .2 & .2 & .2 & & 22.3 & 2.2 & 6.0 & 64.6 & 3.3 & .4 & .3 & .2 & .4 & 50.0 & 26.3 & 12.2 & 6.7 & 3.3 & 0.4 & 2.4 & 642.5 & 644.2 & $\begin{array}{l}679.0 \\
6652.8 \\
637.0 \\
637.0\end{array}$ & $\begin{array}{r}+5.7 \\
8.6 \\
-0.2\end{array}$ & $\begin{array}{l}11 \\
17 \\
0\end{array}$ & $\begin{array}{l}.2 \\
.1 \\
.1\end{array}$ & $\begin{array}{l}16 \mathrm{P} \\
16 \mathrm{P} \\
17 \mathrm{~S}\end{array}$ \\
\hline $\begin{array}{l}17 P \\
17 \mathrm{P} \\
189\end{array}$ & $\begin{array}{l}15700 \\
1377700 \\
1377\end{array}$ & 23.0 & 2.6 & 6.2 & 66.9 & 1.3 & tr & .2 & .2 & .4 & 23.0 & 2.6 & 5.8 & 66.5 & 1.3 & 0 & .4 & .1 & .2 & 53.3 & 25.7 & 11.0 & 7.9 & 1.3 & .4 & 2.4 & 635.5 & 637.2 & $\begin{array}{l}630.5 \\
6393.2 \\
643.2\end{array}$ & $\begin{array}{r}+2.3 \\
+6.0 \\
-0.6\end{array}$ & $\begin{array}{r}5 \\
12 \\
0\end{array}$ & $\begin{array}{l}1 \\
: 1 \\
0\end{array}$ & $\begin{array}{l}17 \mathrm{P} \\
177 \mathrm{Q} \\
178\end{array}$ \\
\hline $\begin{array}{l}18 \mathrm{~S} \\
18 \mathrm{P} \\
18 \mathrm{Q}\end{array}$ & $\begin{array}{l}13772 \\
13773 \\
13774 \\
13774\end{array}$ & 21.7 & 2.7 & 6.1 & 64. 4 & 3.6 & 0.3 & .2 & .1 & $\begin{array}{r}.18 \\
.5 \\
.5\end{array}$ & 21.7 & 2.1 & 5.8 & 63.6 & 3.6 & .5 & .4 & .2 & .8 & 52.0 & 23.0 & 11.8 & 6.4 & 3.6 & .8 & 2.3 & 639.3 & 640.8 & $\begin{array}{l}645.8 \\
649.7 \\
649.7\end{array}$ & $\begin{array}{r}+5.0 \\
8.9 \\
8.9\end{array}$ & $\begin{array}{l}10 \\
18\end{array}$ & $\begin{array}{r}3 \\
3 \\
3 \\
3\end{array}$ & $\begin{array}{l}18 \mathrm{P} \\
18 \mathrm{P} \\
189 \mathrm{Q}\end{array}$ \\
\hline $\begin{array}{l}19 \mathrm{~S} \\
19 \mathrm{P} \\
19 \mathrm{Q}\end{array}$ & $\begin{array}{l}13775 \\
13776 \\
13777\end{array}$ & 22.1 & 2.8 & 5. 5 & 63.4 & 4.7 & .3 & .2 & .1 & & 22.1 & 2.8 & 5.2 & 62.8 & 4.7 & 0 & .3 & .3 & .8 & 48.8 & 26.6 & 9.1 & 8.5 & 4.7 & .5 & 1.9 & 639.2 & 640.9 & $\begin{array}{l}641.1 \\
644.2 \\
646.3 \\
6390\end{array}$ & $\begin{array}{l}0.2 \\
3.3 \\
5.4 \\
1.2\end{array}$ & $\begin{array}{r}0 \\
11 \\
11 \\
2\end{array}$ & $\begin{array}{r}.8 \\
.3 \\
.3 \\
.1\end{array}$ & $\begin{array}{l}19 \mathrm{~S} \\
19 \mathrm{P} \\
190 \mathrm{Q} \\
20 \mathrm{~S}\end{array}$ \\
\hline $\begin{array}{l}20 \mathrm{P} \\
20 \mathrm{P} \\
20 \mathrm{Q}\end{array}$ & $\begin{array}{l}13778 \\
1379 \\
13780\end{array}$ & 19.9 & 3.9 & 7.5 & 66.0 & 1.0 & .6 & .4 & .2 & $\begin{array}{l}1.3 \\
1.4 \\
1.4\end{array}$ & 19.9 & 3.9 & 7.0 & 64. 7 & 1.0 & 0 & .5 & .2 & .8 & 59.5 & 12.2 & 12.0 & 11.9 & 1.0 & 1.3 & 1.9 & 637.8 & 637.8 & $\begin{array}{l}639.0 \\
6344.0 \\
664.0\end{array}$ & $\begin{array}{l}1.2 \\
6.2\end{array}$ & $\begin{array}{r}2 \\
13 \\
13\end{array}$ & $\begin{array}{l}.1 \\
.1 \\
.1\end{array}$ & $\begin{array}{l}20 \mathrm{P} \\
20 \mathrm{P} \\
20 \mathrm{Q}\end{array}$ \\
\hline $\begin{array}{l}21 \mathrm{~S} \\
21 \mathrm{P} \\
21 \mathrm{Q}\end{array}$ & $\begin{array}{l}13781 \\
13782 \\
13783 \\
1372\end{array}$ & 20.9 & 3.0 & 7.6 & 66.0 & 1.3 & .3 & .4 & .2 & $\begin{array}{r}.1 \\
.4 \\
.4\end{array}$ & 20.9 & 3. 0 & 7.2 & 65.6 & 1.3 & 0 & .4 & .4 & .6 & 55.6 & 18.0 & 14.0 & 9.1 & 1.3 & 0.4 & 2.5 & 646.1 & 646.1 & $\begin{array}{l}646.8 \\
648.5 \\
650.6 \\
6441.6\end{array}$ & $\begin{array}{r}2.7 \\
4.5 \\
-2.7\end{array}$ & $\begin{array}{l}1 \\
5 \\
9 \\
0\end{array}$ & $\begin{array}{r}.1 \\
.1 \\
1.1\end{array}$ & $\begin{array}{l}21 \mathrm{P} \\
21 \mathrm{P} \\
21 \mathrm{Q} \\
22 \mathrm{~S}\end{array}$ \\
\hline $\begin{array}{l}22 \mathrm{P} \\
22 \mathrm{P} \\
22 \mathrm{Q}\end{array}$ & $\begin{array}{l}13574 \\
13785 \\
13786 \\
13787\end{array}$ & 21.9 & 2.6 & 6.0 & 63.4 & 4.4 & .2 & .2 & .2 & $\begin{array}{r}.3 \\
.2 \\
\end{array}$ & 21.9 & 2.6 & 5.7 & 63.1 & 4. 4 & 0 & .3 & .5 & .9 & 48.3 & 26.3 & 10.7 & 7.9 & 4.4 & .3 & 2.3 & 642.6 & 644.3 & $\begin{array}{l}641.0 \\
645.6 \\
649.9 \\
699.9\end{array}$ & $\begin{array}{r}+1.3 \\
5.6 \\
5.6\end{array}$ & \begin{tabular}{r|}
0 \\
3 \\
11
\end{tabular} & $\begin{array}{r}0.8 \\
.5 \\
\end{array}$ & $\begin{array}{l}22 \mathrm{P} \\
22 \mathrm{P} \\
22 \mathrm{Q}\end{array}$ \\
\hline $\begin{array}{l}231 \\
23 Q\end{array}$ & $\begin{array}{l}137787 \\
1378 \\
13789\end{array}$ & 19.9 & 2.5 & 7.4 & 65.4 & 3.1 & .4 & .3 & .2 & $\begin{array}{l}2.9 \\
1.0 \\
1.6\end{array}$ & 19.9 & 2.4 & 7.0 & 63.4 & 3.1 & .1 & .4 & .5 & .5 & 56.3 & 14.6 & 14.6 & 7.3 & 3. 1 & 2.0 & 3.0 & 655.7 & 655.7 & $\begin{array}{l}600.0 \\
668.3 \\
661.3\end{array}$ & $\begin{array}{l}2.8 \\
5.6 \\
5.6\end{array}$ & \begin{tabular}{r|}
5 \\
11
\end{tabular} & $\begin{array}{l}8 \\
: 3 \\
.2\end{array}$ & $\begin{array}{l}250 \\
23 P \\
23 Q\end{array}$ \\
\hline
\end{tabular}


subjected to special heat treatments in the laboratory. The special heat treatments, to which the clinker was subjected in thelaboratory, were of such character that different degrees of crystallization of the clinker compounds, and consequently different glass contents might be anticipated.

Factors other than glass content which may cause some slight variations in the heat of solution of the clinker have been discussed in a previous paper [2].

The results obtained from this investigation indicate that, for a clinker of given oxide composition, the heat of solution of the clinker, and likewise the glass content, will be dependent on the cooling conditions to which the clinker has been subjected. The largest heat of solution of a given clinker, that is the highest glass content, was obtained from the rapidly cooled clinker. The slowly cooled clinker always gave a lower heat of solution and was, therefore, of low glass content. In some instances the glass content of the plant clinker as received was of the same order as the minimum glass content of the slowly cooled laboratory clinker. In other cases the glass content of the plant clinker closely approached the maximum glass content obtained in the rapidly cooled laboratory clinker. This seems to indicate that the cooling rates attained in commercial practice vary in sufficient degree to produce large differences in the glass content of commercial clinker.

In a previous investigation [1] it was found that the heat treatment of portland cement clinker has an important effect on some of the properties of the cement. The heat treatments used in that investigation were essentially the same as the special laboratory heat treatments which were used in this investigation and which have now been shown to produce different glass contents in the clinker. Since the commercial products used in this investigation have been found to vary greatly in glass content, it seems probable that the variations in properties of commercial cements, which cannot be accounted for on the basis of composition, may be partly or entirely accounted for from a consideration of their glass content.

Previous investigators $[2,7,8]$ have found that a considerable quantity of $\mathrm{MgO}$ dissolves in the liquid formed at the clinkering temperatures, and when the compositions were cooled rapidly this MgO remained in solution in the glass. The qualitative results of insoluble $\mathrm{MgO}$, as obtained in this study, give further evidence of the solution of $\mathrm{MgO}$ in the glass. For each composition containing 2.4 percent of $\mathrm{MgO}$, or more, the largest percentages of insoluble $\mathrm{MgO}$ were obtained from the slowly cooled clinker, whereas the lowest percentages were obtained from the rapidly cooled clinker.

\section{REFERENCES}

[1] Wm. Lerch and W. C. Taylor. Concrete (CMS) 45, 199 (1937).

[2] Wm. Lerch and L. T. Brownmiller. J. Research NBS 18, 609 (1937) RP997.

[3] Wm. Lerch. Eng. News Record 113, 523 (1934).

[4] R. H. Bogue. Ind. Eng. Chem. Anal. Ed. 1, 192 (1929).

[5] L. A. Dahl. Rock Products. 32, No. 23, 50 (1929).

[6] O. K. Johannson and J. Thorvaldson. J. Am. Chem. Soc. 56, 2327 (1934).

[7] W. C. Hansen. J. Am. Chem. Soc. 50, 3081 (1928).

[8] H. F. McMurdie and F. Insley. J. Research NBS 16, 467 (1936) RP884.

Washingtón, November 17, 1937. 